\title{
Low pressure bottom-up synthesis of metal@oxide and oxide nanoparticles: control of structure and functional properties
}

\section{Sergio D'Addato, Maria Chiara Spadaro}

Dipartimento Scienze Fisiche, Informatiche e Matematiche and En\&Tech, Università di Modena e Reggio Emilia, via G. Campi 213/a, 41125 Modena, Italy

and

CNR-Istituto Nanoscienze, via G Campi 213/a, 41125 Modena, Italy

\begin{abstract}
The experimental activity on core@shell, metal@oxide and oxide nanoparticles (NP) grown with physical synthesis, and more specifically by low pressure gas aggregation sources (LPGAS) is reviewed, through a selection of examples encompassing some potential application in nanotechnology. After an introduction on applications of NP, a brief description of the main characteristics of the growth process of clusters and NP in the LPGAS is given. Successively, some relevant case studies are reported:

- Formation of native oxide shells around the metal cores in core@shell NP.

- Experimental efforts to obtain magnetic stabilization in magnetic core@shell NP by controlling their structure and morphology.

- Recent advancements in NP source design and new techniques of co-deposition, with relevant results in realization of NP with greater variety of functionalities.

- Recent results on reducible oxides NP, with potentialities in nano-catalysis, energy storage and other applications.

Although this list is far from being exhaustive, aim of the authors is to give the reader in a descriptive way some glimpse of the physics behind the growth and the studies of low pressure gasphase synthesized NP, with their ever-growing potentialities for a rational design of new functional materials.
\end{abstract}

Keywords: nanoparticle sources, core@shell nanoparticles, exchange bias, reducible oxides, TEM, XPS, magnetometry 


\section{Introduction.}

Nanotechnology is now part of our everyday life. From electronic goods, to medicine and diagnostics, to food processing and packaging, nanomaterials are widely employed. Out of the many examples, it is possible to single out three types of commercial products employing nanoparticles (NP): the Quantum Dots (QD) televisions, the first types of food package employing Ag NP for antimicrobial purposes, and the contrast agents employed in magnetic resonance imaging, composed of Super Paramagnetic Iron Oxide (SPIO) NP for cancer diagnostic.

In some commercial television screens, nanoparticles of CdSe and other types of QDs are employed as filters in backlighting units of the liquid crystal displays (LCD). It is well known that in colloidal nanoparticles like CdS and CdSe the optical properties of QDs are size dependent: emission and absorption depends on the size $d$ because of quantum size effects [1]. This means that the color of the emitted and absorbed light in a QD can be finely tuned with a proper choice of $\mathrm{d}$, in a range approximately between 2 and $7 \mathrm{~nm}$ (see figure 1). In commercial QD television, radiation from a blue Light Emitting Diode (LED) is transformed by QDs into pure red and green, and the combination of blue, green and red light inhibits absorption of extra tonalities by the filters behind the LCD screen [2]. In this way there is a substantial increase in light throughput and a significant improvement of color gamut. It must be noticed, however, that QD TV are still essentially based on LCD technology. The use of QD as LED, or Q-LED, in displays is still at an experimental stage, although it may potentially be an alternative to the established Organic LED displays [3]. This type of NP can be employed in medical imaging (with proper capping and functionalization to make them biocompatible [4,5]) and, more recently in TV screens. Still, the use of metals like Cd in this type of devices has some limitations because of health issues. As an example, the European Union Restriction of Hazardous Substances Directive, poses severe limits to the use of this type of metals in consumer electronics.

Figure 1. An example of application of NP: Fluorescence spectra of CdSe with quantum dots of different sizes (A), and a picture showing their relative diameter (B). The particle diameters are respectively $2.1 \mathrm{~nm}$, $2.5 \mathrm{~nm}, 2.9 \mathrm{~nm}, 4.7 \mathrm{~nm}$, and $7.5 \mathrm{~nm}$, going from blue to red. Reproduced from [4] with permission of the Royal Society of Chemistry.

The use of silver as antibacterial agent dates back to history. A common method to prolong the freshness of milk was to place a silver spoon or coin at the bottom of the milk container [6]. Recently the food-packaging industry of some countries has introduced products employing nanocomposite material, with nano-silver in containers and coated films. However, these developments raise some questions regarding the possibility of migration of the Engineered NP (ENP) into food and their potential toxicity (see fig. 2) [7]. The investigation of the changes occurring to the physical and chemical properties of materials when passing from micro- to nanoscale are therefore of paramount importance in addressing the many technological and safety challenges posed by the introduction of nanotechnology in food processing and packaging industry [8].

Figure 2. Transmission Electron Microscopy (TEM) image showing the migration of Ag NP detected in an 
orange juice sample stored in nano-silver coated film packaging for 10 days at $40^{\circ} \mathrm{C}$. The length of the scale bar is $6 \mathrm{~nm}$. Reproduced from [7] with permission from Elsevier.

A third example that is worth to be mentioned is taken from the field of medicine and diagnostic, in particular the employment of ENP as contrasting agent in magnetic resonance imaging (MRI) for diagnostic of cancer in liver and other internal organs [8]. Super-Paramagnetic Iron Oxide Nanoparticles (SPION) have been used for the last thirty years in MRI for detection of focal liver and spleen lesions. Their diameter is generally approximately $30 \mathrm{~nm}$, but more recently ultrasmall SPION (U-SPION) have been in use, with their size significantly smaller. SPIONs are typically composed of magnetite $\left(\mathrm{Fe}_{3} \mathrm{O}_{4}\right)$ or maghemite $\left(\gamma-\mathrm{Fe}_{2} \mathrm{O}_{4}\right)$ single crystals. Other NP that can be potentially used in MRI are Co, core@shell Au@Fe and Pt@Fe. The possibility of producing core@shell NP have extended the potentiality of applications in this field [9], with significant enhancement in the diagnostic and therapeutic capabilities.

The huge advancements in NP synthesis and functionalization have allowed their use in many industrial products, but the requirements for a control of their properties, especially regarding their effects on human health and environment is becoming more and more stringent. The crucial problem, as mentioned before, is the understanding of the modification of the physical and chemical properties of matter when passing from the micro- to the nano-scale. The effects on the structure of metal NP can be given as an example. It is well known that in small, stable metal clusters ${ }^{1}$ with $d$ of few $\mathrm{nm}$, atoms are arranged in closed geometric shells [10]. In fact, clusters do not possess translational symmetry like "bulk" crystals. However, in a conceptual bottom-up process, we can imagine building the particle by forming polyhedral concentric shells of atoms, in a sort of "shell periodicity"; the concentric layers of atoms are added in a fashion that the overall symmetry of the clusters does not change. Within this approach, when the shell of atoms is complete, the cluster has a stable configuration because the average atomic coordination number is maximized. Experimentally, this gives rise to the occurrence of "magic numbers", i.e. sets of numbers of atoms for which clusters are energetically stable. For instance, in alkali metal clusters, the magic numbers are those obtainable by completing $K$ polyhedral shells, based on 12 -vertex icosahedra. This would result in particles with the shape of icosahedron (see fig. 3), Ino decahedron and face centered cubic cuboctahedron [11]. At increasing size, these types of structure can become metastable (i.e. they do not correspond to the absolute minimum of the free energy), but they can still be observed, if the NP are generated using gas phase physical synthesis processes [12-15]. In a recent wide angle Xray scattering experiment with pulsed free electron laser radiation, scattering patterns from in-flight single Ag NP could be detected [16]. The most relevant result was the observation of patterns typical of metastable NP, even for very large sizes $\left(\approx 300 \mathrm{~nm}, N \approx 10^{9}\right.$ atoms $)$. It can be predicted that these states (in many cases with icosahedral and decahedral shapes) show a phase transition to fcc-derived structures. Their occurrence for larger size NP (with $N \approx 10^{6}$ atoms) was ascribed to non-equilibrium growth process in the NP source from small seeds. Large NP with a highly oblate shape could also be singled out, suggesting that the seed structure "locks" the growth mode, in analogy to formation of snowflakes.

\footnotetext{
1 We will adopt the term "cluster" for agglomerate of atoms with size $N<500$, while the term "nanoparticle" will be used for bigger size values. This choice is of course completely arbitrary, but it is convenient when the first stage of growth of NP is discussed. Moreover, many sources are also called cluster or nanocluster sources.
} 
Figure 3. Icosahedral structure for a metal NP. Picture courtesy of V. Grillo.

The morphology and the surface structure of the NP can influence the properties of functional molecules absorbed on the exposed facets, or can determine some interfacial effects, like exchange bias (EB) occurring in ferrromagnetic-antiferromagnetic (FM-AF) material interfaces, like in the case of core@shell configurations [17], so new architectures and functionalities can be designed. In most of applications, NP are produced by chemical or green synthesis. These methods are generally scalable for industrial production. Nevertheless, because of their commercial applications and of potential extended use in many fields, characterization and in-deep investigation of the "case study" NP are necessary, for a fine tuning of the desired properties. Physical synthesis, bottom-up techniques have the advantage of "reduced" complications due to absence of chemical by-products and ligands, and the possibility of one-step processing. The bottom-up approach allows a precise control in the formation of NP and of its characteristics. Aim of this review is to focalize on some specific examples of growth and investigation of low pressure gas phase synthesized NP, in order to demonstrate the potentiality of this research in realizing significant advances in nanotechnology. The paper is organized as follows: a brief summary of the principal techniques used for gas phase synthesis of NP will be given. Successively, some selected experiments, with realization and studies of metal@oxide and oxide NP will be described, with an accent on the interplay between their structure and functional properties: tuning of exchange bias in FM/AF systems, reversible reducibility and oxidation in oxide NP. Finally, some conclusions and future perspectives will be given.

\section{Physical synthesis of nanoparticles with a bottom-up approach}

Physical synthesis of NP using a bottom-up approach has a long tradition in physics and chemistry of materials. The basic principle is to form small aggregates of atoms that can grow by attachments of single atoms, coagulation or coalescence. The resulting clusters can be free particles in a "cloud", in a collimated beam, or they can be adsorbed on a support. A variety of clusters and nanoparticles sources have been successfully developed. It is worth to mention the seeded supersonic cluster source, the laser ablation source [18-19], the pulsed-arc cluster ion source and pulsed microplasma cluster source [18-20]. These sources generally operate with low pressure gas carrier. Recently, great advancements have been obtained in gas-phase synthesis with other methods, like flame-based synthesis [22], non-thermal plasma based synthesis [22-25], which have been capable to produce core-shell nanoparticles [26] and spark discharge-based synthesis [27], extending the possibilities in realizing scalable physical synthesis methods for producing nanomaterials. In this review we will focus our attention on the experiments performed with Low Pressure Gas Aggregation Sources (LPGAS), like the one shown in figure $4[28,29]$.

Figure 4. One of the first examples of gas aggregation source [28,29]. Reproduced from [29] with permission from Elsevier.

In LPGAS, atoms are generally evaporated from a furnace, or by sputtering from a target. The vapor is cooled by interaction with atoms of a noble gas (typically Ar) at low temperature, and 
clusters start to grow in the so-called gas aggregation region (condensation region in figure 4). The noble gas acts also as a gas carrier, and the beam of clusters/NP exit the gas aggregation region through an aperture into a second chamber with a higher vacuum. The NP/gas carrier beam can therefore be formed because of differential pressure between the gas aggregation region and the second chamber. In a variant method, a pulsed laser is used ablate material from a targe and to inject it in a pulsed gas stream. This arrangement produces a pulsed cluster beam [30]. Another possibility of growing NP can occur when a beam of atoms from a thermal source impinges on a surface. Nano-islands can grow spontaneously or after thermal treatment. In this case the NP will be formed directly on a substrate [31-33].

Figure 5 a) Scheme of a magnetron head for sputtering. Insets: Argon-electron collisions give rise to ionization. This process reaches its maximum efficiency when the magnetic field lines lie parallel to the surface. Argon ion bombards the target surface, ejecting atoms and electron. Reproduced from [36] with permission by John Wiley and Sons. b) Scheme of the magnetron sputtering gas aggregation source designed by H. Haberland [34].

We pass now to a more detailed description of sputter gas aggregation sources, based on the original design of Haberland [34-36], which is commonly used by many groups. As mentioned above, metal atoms are evaporated by sputtering of a target using a D.C. magnetron. A magnetron consists essentially of:

- A cylindrical electrode (cathode) on which a disk-shaped target of the material is attached. The cathode is set to a negative voltage of few hundred volts (100-300 V)

- An anode set to ground, in the shape of a ring surrounding the cathode. The distance between the two electrodes is about 0.3-0.5 mm. Both electrodes are in vacuum.

- A permanent magnet positioned behind the magnetron.

The system is also cooled, either with liquid nitrogen or water. In operating conditions, Ar gas flows between the electrodes, giving rise to a glow discharge. Ar atoms get positively ionized and bombard the target, ejecting atoms and electrons out of the material. The electrons giving origin to the discharge are confined by the magnetic field to a region where magnetic field lines are parallel to the target surface. In this way the sputtering rate is enhanced, as more Ar atoms are positively ionized. The glow discharge, because of the magnetic field line distribution, has an annular shape, and the target gets eroded in a circular region. The sputtered atoms can be charged or neutral. While the neutral atoms escape the glow discharge region and form the clusters, the positively ionized sputtered atom are deposited again on the target surface. After leaving the discharge regions, the small clusters start forming and increase their size, as they travel along the aggregation region with the gas carrier, that can be composed of $\mathrm{Ar}$ or a mixture of different species: $\mathrm{He}, \mathrm{Ne}, \mathrm{N}_{2}$ but also $\mathrm{O}_{2}$, depending on the type of NPs to be realized. It is generally accepted that condensation starts with the formation of dimers and it goes on by adding single neutral metal atoms [37-40]:

$\mathrm{M}+\mathrm{M}+\mathrm{Ar}->\mathrm{M}_{2}+\mathrm{Ar}$ 
However, in a recent paper by Haberland [36], it is argued that condensation starts first with formation of $\mathrm{Ar}^{+}$, which is given by

$\mathrm{Ar}^{+}+\mathrm{Ar}+\mathrm{Ar}->\mathrm{Ar}_{2}^{+}+\mathrm{Ar}$

and then it continues with subsequent attachment of $\mathrm{M}$ atoms, although a detailed description of the initial stages of aggregation remains unknown. Also, some dimers can be formed by a single sputtering process, and can act as condensation germs.

Once the homogeneous nucleation has lead to the formation of "embryos", condensation occurs and (meta)stable NP are formed, which can grow by single atoms attachment, coalescence or coagulation. As mentioned above, the growth kinetics determines the structure and morphology of the resulting NP. Up to now, we summarized the process leading to formation of metal NP by using DC magnetron sputtering. However, RF magnetron sputtering can be used in the case of NP composed of non-conductive materials. The magnetron sputtering gas aggregation source can produce beams of NP in a very extended range of size, from few ( $d$ less than $1 \mathrm{~nm}$ ) to several hundred of thousands of atoms, corresponding to $d$ greater than $30 \mathrm{~nm}$. Also, the size distribution can be quite narrow, with a Full Width Half Maximum in the mass distribution course of about 20$30 \%$. The shape of the size distribution is generally well represented by the log-normal distribution, which was theoretically predicted by simulation of the aggregation processes using the MonteCarlo technique $[41,42]$. The experimental conditions affecting the characteristics of the NP generated by the magnetron sputtering gas aggregation source are determined by the following parameters:

- Ar gas flow in the discharge region and in the aggregation regions, which can be adjusted with mass flow controllers or with adjustable leak valves.

- Discharge power.

- Length of the gas aggregation tube, i.e. the travel length of the cluster during the process of aggregation and growth.

Additionally, a mixture of gas in the aggregation region can affect the size distribution: for instance, $\mathrm{He}$ is used for producing smaller clusters and NP. In some experiments, $\mathrm{O}_{2}$ was flown to generate oxide NP $[43,44]$.

Once the NP beam exits the LPGAS, the size distribution can be analyzed with mass spectra. Quadrupole Mass Spectrometers/Filters (QMF) or Time of Flight Spectrometers (TOFS) are generally used [29]. The QMF can select and measure the ionized NP, which can be present in the beam in a consistent fraction (for some instruments, up to 80\%). After this stage, the NP can be deposited on a support and investigated with a variety of techniques, in situ or ex situ. In addition, if the NP are electrically charged, their impact energy can be varied with the help of an electrostatic field, for instance by setting the support at high voltage. Some important studies on the effect of impact energy on the NP morphology and structure when deposited on a substrates were performed experimentally [34, 35] and with Molecular Dynamics simulations on Mo clusters deposited on $\mathrm{Cu}$ and Mo substrates [45], revealing three different regimes:

- If the kinetic energy of the cluster just before the impact onto the surface is $E_{k}<1 \mathrm{eV} /$ atom (low energy regime) the cluster structure is only slightly deformed (soft landing).

- If $\mathrm{E}_{\mathrm{K}}$ lies between 1 and $10 \mathrm{eV} /$ atom (medium energy) the morphology of the cluster can change and there may be some induced defects on the support.

- If $\mathrm{E}_{\mathrm{k}}>10 \mathrm{eV} / \mathrm{atom}$ the cluster is disrupted, the substrate is damaged in the impact area, and the damage can extend down to several atomic layers. 
During normal operation, the magnetron based LPGAS generate beams of NP at low energies, although some deformation of NP after landing was observed. This change of shape (a sort of slight "flattening") was ascribed to electrostatic interaction between the NP and the substrate [46].

There are additional possibilities for more complex NP architectures, finalized to realization of core@shell, dumb-bell geometries, nanoalloys etc. [47-50]. Also, chemisorption of functional molecules on in-flight NP or growth of NP in solid matrix and/or multilayered NP films are possible, with some implementation of the equipment. Some examples will be discussed in the following section. In figure 4 a sketch of a typical experimental apparatus equipped with a NP source is shown. The system, designed and used by our group [15, 46], consists of four interconnected ultra-high vacuum chambers:

- The NP source and the QMF, where the NP beam is generated and mass-filtered.

- The deposition chamber where the substrate is inserted through a fast entry arm and positioned across the NP beam. In this chamber there are also evaporators for capping and/or co-deposition treatment, a gas-line for letting in $\mathrm{O}_{2}$ and other gas species, a quartz microbalance for monitoring the amount of the deposited material and an oven for thermal treatment.

- Two analysis chambers where the NP film deposited on the substrate is transferred for X-ray Photoelectron Spectroscopy (XPS) and Auger Electron Spectroscopy analysis.

XPS is mostly used, for an in situ chemical analysis of the produced NP film. Other techniques are employed ex-situ; for instance Scanning Electron Microscopy (SEM), atomic force microscopy, Transmission Electron Microscopy (TEM), High Resolution TEM and Scanning TEM, to determine the structure and morphology, but a great number of experiments can be performed, depending on the properties and on the functions of the NP that need to be investigated.

Figure 6. Sketch of the experimental apparatus used in the work described in $[15,46]$, with the NP source and QMF filter, deposition chamber and XPS and Auger electron spectroscopy chambers for NP film analysis.

\section{Low pressure gas phase synthesis of core@shell nanoparticles: some selected examples}

\subsection{Native oxide shells in metal nanoparticles}

The investigation of metal clusters and NP produced by low pressure gas phase synthesis is a vast subject, that has been carried out for the last five decades. Structure, electronic, magnetic and optical properties have been studied in deep. The reader is referred to some recent books and excellent reviews [29, 51-53]. This section is dedicated to the activities on core@shell NP, as this configuration presents interesting opportunities for nanomaterial functionalities. The simplest way to obtain a shell around a metal NP is to expose it to atmosphere. The surface of the NP will be oxidized, and the result will be a NP with a metallic core and a shell of native oxide. In a strict sense, most of the metal NP exposed to atmosphere (even for a short time) while they are transferred to a different apparatus, like for instance a TEM, have a shell of native oxide; some notable exceptions are Au NP, or core@shell NP with an Au shell [40]. The thickness of the native oxide shell will depend on different factors, like the exposure time and the NP reactivity to air exposure. The shell can modify the original properties of the NP. The structure and physical properties of the external layer will change, as a new material is now formed, as well as the NP morphology and size. Some specific examples of this effect have been studied on Fe [54], Co [55], 
FePt [56], FeCo [57] and Ni [15] NP. In the case of Fe [54], TEM image shown a regular shell, with a thickness $\mathrm{t} \approx 2 \mathrm{~nm}$ over a core diameter of size $\mathrm{d} \approx 5 \mathrm{~nm}$ (see fig. 7 ).

Figure 7. TEM image of a Fe NP after exposure to air, where the oxide shell is clearly visible. The lattice fringe spacing of the core corresponds to $<110>$ planes of $\alpha$-iron. Reproduced from [54], with the permission of AIP Publishing.

In Co NP of diameter $\mathrm{d} \approx 8 \mathrm{~nm}$, a value of $t \approx 1 \mathrm{~nm}$ was obtained for the native CoO. For smaller sizes $(\mathrm{d} \approx 2 \mathrm{~nm})$ the NP was reported to be completely oxidized [55]. The effect of exposure to atmosphere and controlled oxidation of Ni NP of size d between 4 and $8 \mathrm{~nm}$ was systematically investigated with XPS and TEM [15]. The Ni NP were deposited on inert substrates (namely, Si wafers with their native oxide $\mathrm{Si} / \mathrm{SiO}_{\mathrm{x}}$ and $\mathrm{HOPG}$ ), and were oxidized with different procedures: co-deposition in presence of $\mathrm{O}_{2}$, exposure to $\mathrm{O}_{2}$ after deposition, exposure to atmosphere for 12 hours, exposure for 20 days. The treated NP assemblies were then analyzed with XPS (see fig. 8a), focusing on the photoelectron spectra from Ni $2 p$ core levels. The spectra were deconvolved in two chemical-shifted components, corresponding to emission of electron from $\mathrm{Ni}$ in a metallic state and from $\mathrm{Ni}$ in an oxidized state (see fig. 8b). The relative intensities of the two components allowed to estimate a thickness for the oxide shell of no more than few atomic layers after 12 hours of exposure. Moreover, a metallic Ni core was still present after more that 20 days of air exposure.

Figure 8. a) Ni $2 p$ XPS spectra from Ni NP on HOPG taken after different oxidation stages. Step 1; spectrum taken immediately after NP deposition. Steps 2-5: data taken after subsequent stages of oxidation. At the bottom is reported the Ni $2 p$ spectrum from NiO. b) Red line: Ni $2 p$ spectra taken from Ni NP at step 2 and step 4 with relative components: metallic Ni (black) and oxidized (blue) component. The oxidized component was obtained after subtracting a Shirley background and the metallic component (corresponding to the spectrum taken at step 1) from the original data. (C) IOP publishing. Reproduced with permission from [15]. All rights reserved.

Similarly to many other metal NP realized with low pressure gas phase synthesis, HR-TEM images taken from Ni NP deposited on TEM grids revealed a multitwinned icosahedral structure for the metallic core [12-14]. Local strain analysis showed also deformation of the peripheral Ni region which can be associated to growth of epitaxial NiO crystalline islands on the NP surface. The overall behavior was consistent with a picture often given by surface oxidation of metals consisting of three phases:

1. Dissociation of oxygen molecules and chemisorption of oxygen atoms on the metal surface.

2. Formation of $\mathrm{NiO}$ islands and their nucleation into a continuous $\mathrm{NiO}$ layer.

3. Growth of the NiO film.

The velocity of the third process depends strongly on the temperature and is hampered by the limited diffusion of the nickel cations through the oxide film. For temperatures below 500-600 $\mathrm{K}[58,59]$, like in this case, the number of oxide layers that can be actually grown is limited. After their formation, they passivate the metallic nickel surface and stop the increase of the oxide film thickness. 
Figure 9. a) HRTEM image of a metallic Ni NP. b) Fourier Transform (FT) of image in a. c) FT of the amorphous region around the NP and its relative fit. d) Simulation of an image from a Mackay crystal with 15 shells. e) model of the Mackay icosahedral crystal structure, f) FT of the model reported in e). (C) IOP publishing. Reproduced with permission from [15]. All rights reserved.

A more recent investigation on the process Fe NP oxidation was carried out with aberrationcorrected STEM [60]. In this case, cuboid-shaped Fe NP of width 5-20 nm were gas-phase synthesized and imaged after prolonged times of exposure to air, even after two years. A core-shell geometry was observed after six months deposition, with a shell showing an inverse spinel structure composed by $\mathrm{Fe}_{3} \mathrm{O}_{4}$ and $\gamma-\mathrm{Fe}_{2} \mathrm{O}_{3}$ phase. Interestingly, no equilibrium oxide thickness (which was estimated to be 2-3 nm in Cabrera-Mott theory of metal oxidation [61]) is reached. After approximately 2 years, the particles were fully oxidized. The strain analysis of STEM images explained the complete oxidation (and the presence of Kinkerdall voids at the centre of the NP [62]) as due to positive strain gradients leading to increased ionic conductivity. As a result, there is diffusion of cations towards the NP surface, occurring by exchange of vacancies, and a diffusion of anion through the interstitial sites. Oxidation process in these NP does not stop, as in the case of bulk metals. The reason given by the authors is the impossibility of creating dislocations that allows relaxation of the elastic strain at the metal-oxide interface, which prevents the oxidation to further progress. The different behavior between the two reported cases (Ni and Fe NP) can be probably ascribed to the different NP shape and structure.

Figure 10. a-c, Aberration-corrected STEM image of Fe NP taken after 6 (a), 12 (b) and 24 (c) months of exposure to atmosphere. The dashed lines highlight the front of oxidation, moving towards the inner core of the NP. In c), the NP is completely oxidized, with the evidence of formation of four domains. $\mathbf{d}$, line profile of the dashed region evidenced in c, showing a Kirkendall void at the center of the NP [60]. Reproduced by permission from Macmillan Publishers Ltd: Nature Materials [60], copyright (2013).

\subsection{Magnetic properties of metal@oxide NP: exchange bias}

As already mentioned, creation of an oxide shell can have major effects on the electronic and magnetic properties of the NP. A typical example is given by metal@oxide NP with a ferromagnetic (FM) core and an antiferromagnetic (AF) shell, like in $\mathrm{Co@CoO}$ and Ni@NiO NP. It is well known that FM bulk materials show magnetic domains, as a consequence of energy minimization [63]. In fact, the competition between magnetostatic energy and the energy associated to formation of domain walls favors the presence of domains. Nevertheless, when the finite size of the NP decreases below a certain limit, formation of multiple domains is not energetically convenient anymore, as the total energy must be minimized, including the magnetostatic energy, the exchange and the anisotropic terms as well as the domain contribution. In this case NP can exhibit a single magnetic domain, with all the atomic spins oriented along a certain preferred direction, depending on particle shape and anisotropy. The NP can be considered in this case as a single "giant" spin. The transition from multi-domain to single domain regime in small particles was studied theoretically by C. Kittel [64]. He estimated a value for the critical size of a small particle of 
the order of $10 \mathrm{~nm}$. It was found that the critical size can vary between 20 and $800 \mathrm{~nm}$. This value depends on the energies of anisotropy and of exchange. In Fe spherical NP, for instance, the transition from multi- to single domain occurs with particles of diameter of about $30 \mathrm{~nm}$, while for Co the critical size is typically $35 \mathrm{~nm}$ [65]. As a consequence, an assembly of single domain NP can be conceived as an ensemble of magnetic dipoles, and inside the NP the atomic spins will be subject to coherent rotation. The giant spin of free NP will depend, of course on the NP size, but also on its shape and structure. A series of experiments on beams of clusters/NP were performed by a number of groups, aimed at the measurements of their magnetic moment [66]. Some of these works followed the conceptual idea of the Stern-Gerlach experiments, i.e. a beam of mass selected clusters or small NP were deflected by the force exerted by the gradient of a magnetic field. The deflection was recorded generally with a Time of Flight (TOF) Mass Spectrometer. By knowing the field gradient $d B / d z$ and other quantities like the length of the magnet $L$ and the length of the flight tube between the magnet and the mass spectrometer $D$, the magnetic moment per atom, $m$, of the sizeselected clusters could be obtained [67-75]. Another important technique used for determining the magnetic moment per atom of free and supported NP is X-ray Magnetic Circular Dichroism in absorption spectra (XMCD), where synchrotron radiation X-rays are employed [86-78]. The knowledge of NP magnetic properties is crucial for the design of advanced magnetic memories, in particular regarding the need of high density storage devices. To this purpose, the miniaturization of the single element of memory can be hampered by the loss of magnetic stability due to thermal fluctuations [79].

Figure 11. Onset of superparamagnetism in a single domain NP. If the diameter of the NP $D$ is sufficiently small, the anisotropy energy $E_{a}=K V$ will be comparable with thermal energy and the particle will start to oscillate at high frequency between the two energy minima, resulting in magnetic instability. Picture courtesy of A. Ponti.

In a small magnetic particle, the existence of a certain uniaxial magnetic anisotropy will give rise to a preferred orientation of its magnetic moment along an easy axis of magnetization. In order to reverse the orientation of this moment, an energy barrier (called anisotropy energy) $\Delta \mathrm{E}=\mathrm{K}_{\mathrm{a}} \mathrm{V}$ has to be overcome, where $\mathrm{K}_{\mathrm{a}}$ is the anisotropy constant and $\mathrm{V}$ is the particle volume. When $\mathrm{V}$ is reduced, the energy barrier is lowered, and the NP thermal energy $\mathrm{K}_{\mathrm{b}} \mathrm{T}$ can give rise to fluctuations more and more frequent that can overcome the anisotropy barrier. In this case the NP magnetic moment will not be blocked anymore, and an assembly of NP showing this behavior will be in a superparamagnetic phase. On the contrary, if the NP remain stable during a certain measurement time, $t_{m}$, it is said that the NP are blocked. The blocking temperature, conventionally defined by the formula $\mathrm{T}_{\mathrm{B}}=\mathrm{K}_{\mathrm{a}} \mathrm{V} /\left(25 \mathrm{~K}_{\mathrm{B}}\right)$, corresponds to the temperature of transition from blocking to superparamagnetic phase [65]. $\mathrm{T}_{\mathrm{B}}$ therefore depends of the product between the NP volume and the anisotropy constant. For Ni, given an anisotropy constant $K=0.5 \cdot 10^{4} \mathrm{~J} / \mathrm{m}^{3}$, it can be obtained that $\mathrm{T}_{\mathrm{B}}$ is equal to room temperature (RT) for a NP volume $\mathrm{V}_{\text {crit }}=2.1 \cdot 10^{4} \mathrm{~nm}^{3}$. If the NP is assumed to have a spherical shape, this volume corresponds to a diameter $\mathrm{d} \approx 34 \mathrm{~nm}$. The "superparamagnetic limit" can be therefore a serious obstacle to design and realization of ultrahigh density magnetic memories [79]. 
Figure 12. a) Schematic diagram of a FM/AF spin coupling at a FM/AF layered interface before and after field cooling. b) Main effects of the EB: shift of the hysteresis loop $\left(\mathrm{H}_{\mathrm{E}}\right)$, increase of coercivity and change from uniaxial to unidirectional anisotropy. Reproduced from [80] with permission from Elsevier.

During the last decade, the possibility of obtaining stable magnetic NP has been investigated by exploiting the exchange bias (EB) effect at the interface between a FM and an AF material. In the case of NP, this effect would be obtained by realizing a core@shell configuration, where the core is FM and the shell is AF. Co@CoO, Ni@NiO and Ni@CoO NP should present this effect, as NiO and $\mathrm{CoO}$ are both AF materials. The origin and the explanation of the EB effect still present some difficulties [80]. A first, simple explanation for EB effect occurring at FM/AF planar interface, like in the case of films and multilayers, lies in the additional torque exerted by the exchange coupling between the FM and AF spins when there is a switching of the external magnetic field [80]. Let us suppose that the Neel temperature of the AF material, $T_{N}$, is lower than the Curie Temperature, $T_{C}$, of the FM material, $\mathrm{T}_{\mathrm{N}}<\mathrm{T}_{\mathrm{C}}$. When the system is cooled below $\mathrm{T}_{\mathrm{C}}$ in presence of a magnetic field $\mathbf{H}$ with enough large intensity, all the spins of the FM material are aligned along $\mathbf{H}$. If the temperature is further cooled down below $\mathrm{T}_{\mathrm{N}}$, the spin interaction at the FM/AF interface forces the first layer of spins of the AF material to be aligned along the direction parallel to the spins of the FM medium. This will give rise to an AF ordering with spins parallel or antiparallel to the magnetization $\mathrm{M}$ of the FM. The FM/AF spin interface coupling gives rise to an extra torque exerted on the FM spins, which has to be overcome by $\mathbf{H}$. On the basis of these simple considerations, if the AF/FM coupling is strong enough, there is a rigid shift of the hysteresis loop, while in the case of small AF/FM interaction the result is an increase of the coercition field, i.e., there is some hardening of the magnetic material. Very often, both phenomena occur, because of inhomogeneity of the AF anisotropy due to grain size distribution and structural defects. EB can therefore lead to stabilization of magnetic NP, if the magnetic core is either surrounded by and AF shell or embedded in an AF medium. This behavior was directly verified on chemically and physically synthesized core-shell NP. In particular, low pressure gas phase synthesized NP permitted great flexibility in realizing oxide shells, either by simple oxidation [15, 81], or by co-deposition of NP core and shell [82]. Moreover shells of different oxide species could be realized [48,82]. For instance, in a seminal work [83] assemblies of $\mathrm{Co} @ \mathrm{CoO} N P$ embedded in an AF matrix $(\mathrm{CoO})$ or paramagnetic $\left(\mathrm{Al}_{2} \mathrm{O}_{3}\right)$ matrix were successfully produced with a LPGAS and a conventional sputtering apparatus; structure, composition and magnetic properties were investigated with HR-TEM and with Superconducting QUantum Interference Device (SQUID) magnetometry, obtaining Field Cooled/Zero Field Cooled (FC/ZFC) curves and hysteresis cycles at different temperature values (see figure 13).

Figure 13. a) Sketch of the Co@CoO NP layered films embedded in a matrix. b) Magnetic moments of Co@CoO NP vs Temperature in Field Cooling and in zero field cooling conditions (FC/ZFC curves). Open symbols are ZFC measurements results, closed symbols are FC measurements results. Circles corresponds to measurements from $\mathrm{Co} @ \mathrm{CoO}$ embedded in $\mathrm{AF}$ matrix, diamonds to measurements from $\mathrm{Co} @ \mathrm{CoO}$ embedded in $\mathrm{Al}_{2} \mathrm{O}_{3}$. The measurements from the $\mathrm{NP}$ in $\mathrm{Al}_{2} \mathrm{O}_{3}$ correspond to a superparamagnetic sample with a blocking temperature $\mathrm{T}_{\mathrm{B}}=10 \mathrm{~K}$. The Measurements for NP in $\mathrm{CoO}$ matrix show that Co cores remain FM up to Néel temperature Reproduced by permission from Macmillan Publishers Ltd: Nature [83], copyright (2003). 
The results demonstrated that FM NP embedded in an AF matrix can lead to magnetic stabilization and a substantial increase of $\mathrm{T}_{\mathrm{B}}$, even for Co NP as small as $d=3 \mathrm{~nm}$.

Similar studies were carried out by the same group on $\mathrm{Co} @ \mathrm{CoO} N P$ in $\mathrm{Al}_{2} \mathrm{O}_{3}$, with increasing NP density, revealing a significant increase of $\mathrm{T}_{\mathrm{B}}$, of the coercition and of the bias fields [84]. This effect was ascribed to the "recovery" of the AF properties in the $\mathrm{CoO}$ shells when they come into contact, rather than to interaction between the NP cores. Ni@NiO NP presents some intriguing, different properties with respect to $\mathrm{Co} @ \mathrm{CoO}$. Although the FM anisotropy constant of $\mathrm{Ni}\left(\mathrm{K}_{1}=-5 \mathrm{x}\right.$ $\left.10^{3} \mathrm{~J} / \mathrm{m}^{3}\right)$ and the AF anisotropy constants of $\mathrm{NiO}\left(\mathrm{K}=-500 \mathrm{~J} / \mathrm{m}^{3}\right)$ are significantly smaller than the ones of Co $\left(\mathrm{K}_{1}=45 \times 10^{4} \mathrm{~J} / \mathrm{m}^{3}\right)$ and of $\mathrm{CoO}\left(2.7 \times 10^{7}\right)$, for $\mathrm{CoO} \mathrm{T}_{\mathrm{N}}=291 \mathrm{~K}$, a values which is lower than the one of $\mathrm{Ni}\left(\mathrm{T}_{\mathrm{N}}=525 \mathrm{~K}\right)$, with potential problems in magnetic devices operating at room temperature. Several studies have been carried out on Ni@NiO NP [85-87]. The results on EB and stabilization are sometime controversial. This puzzling behavior can be ascribed to the differences in NP synthesis procedure, which can influence many factors (presence of interface defects and/or dislocations, crystal quality in core and shell, etc). In a recent comparative work, the oxide shell was formed by using different methods, namely exposition to oxygen after NP growth and deposition on substrates, annealing in atmosphere, and the "sequential layer deposition" procedure. $[88,89]$. The sequential deposition method consisted of three phases:

1) $\mathrm{A} \mathrm{NiO}$ layer was grown on $\mathrm{Si} / \mathrm{SiO}_{x}$, by means of thermal evaporation of atomic Ni in presence of oxygen.

2) A beam of metal NPs was generated by LPGAS and impinged on the ultrathin NiO layer.

3) An additional oxide film was deposited on the Ni NP assembly, with the same method used in step 1.

This procedure permits growth of oxide shells with variable thickness, as the Ni core diameter remains fixed, allowing in this way considerable simplification of the analysis. The morphology of the samples prepared with sequential layer deposition was analyzed with STEM in High Angle Annular Dark Field (HAADF) mode and HR-TEM from NP assemblies deposited on carbon-coated copper grids. From the image of a NP shown in figure 14a, there is evidence of a core-shell morphology, where the shell can be assigned to $\mathrm{NiO}$, while the core, which from the contrast appears heavier, is composed of Ni. It is deduced that NiO grows as a shell around the Ni NP synthesized by the LPGAS. The STEM-HAADF image has a shape compatible with a McKay icosahedron. The icosahedral structure is due to multiple twinning of Ni small single crystal exposing (111) facets, a common phenomenon occurring in the fcc metal NP (see also fig. 9).

Figure 14 a) STEM-HAADF image of $\mathrm{Ni@NiO} \mathrm{NP} \mathrm{obtained} \mathrm{with} \mathrm{sequential} \mathrm{layer} \mathrm{deposition.} \mathrm{b)} \mathrm{SEM}$ image of Ni@NiO NP films with a thick $(\mathrm{t}=6.5 \mathrm{~nm})$ overlayer of NiO. c) EWR TEM image of the Ni/NiO interface of Ni@NiO NP synthesized with the same method. d) Simulation of the image shown in c), with the orientation of $\mathrm{NiO}$ shell grown on $\mathrm{Ni}$ (111) facet. (C) IOP publishing. Reproduced with permission from [89]. All rights reserved.

The evolution of the NP film morphology at increasing thickness of the NiO overlayer was investigated with SEM. As the thickness of the NiO overlayer increases, the NP gets bigger, with the presence of small structures that can be ascribed to nucleation of $\mathrm{NiO}$ islands. When the thickness of the $\mathrm{NiO}$ overlayer reaches the value of $\mathrm{t}_{\mathrm{NiO}}=6.5 \mathrm{~nm}$, grains of size up to $60 \mathrm{~nm}$ are 
observed (figure 14b), as a fractured film is formed. Therefore, it can be concluded that at increasing thickness of the overlayer, the NiO shells around the Ni NPs extend progressively, and eventually the Ni NP cores are embedded in a solid matrix. A closer inspection at the core-shell interface structure has been made possible by STEM and HR-TEM, comparing two possible ways of obtaining the NiO: annealing in air of the metallic $\mathrm{NP}$ at $\mathrm{T}=250^{\circ} \mathrm{C}$ for $\mathrm{t}=30 \mathrm{~min}$ and sequential layer deposition. In synthesis, in the first case, the shell has a structure of crystalline $\mathrm{NiO}$, growing in the [111] direction. A more detailed study of the core-shell interface with the Geometrical Phase Analysis of the STEM images put in evidence the formation of dislocation, that probably relax the stress in the interface region, caused by the lattice mismatch between the crystalline fcc $\mathrm{Ni}$ and rock-salt $\mathrm{NiO}$ (111) planes, favoring crystal ordering in the NiO shells. This relaxation and the formation of dislocations can be ascribed to thermal annealing. In the case of the samples prepared with the sequential layer deposition, the interface sharpness is reduced and the shell atomic structure is less ordered. The Exit Wave Reconstruction analysis of HR-TEM images in some parts of the shell shows atomic fringes corresponding to a [243] direction for the NiO growth, different from the original (200) $\mathrm{NiO}$ planes observed for the annealed sample (see fig. 14). In this way, the 3:4 NiO/Ni interface coincidence observed by TEM, which requires an expansion of $11 \%$ in the $\mathrm{NiO}$ lattice, can be explained. The reduced sharpness compared to the annealed sample is ascribed to the two different procedures of realization of $\mathrm{NiO}$ shells. Nevertheless, with the sequential layer deposition it is possible to change shell thickness without varying the core diameter, with a thorough analysis of the effect of the NiO shell thickness on the NP magnetic properties. In practice, EB effect, which is indeed observed with SQUID magnetometry, can be finely tuned by adjusting the shell thickness as a single parameter.

Figure 15. Left: stack of FC/ZFC curves from Ni@NiO NP fils grown with sequential layer deposition, at increasing values of the $\mathrm{NiO}$ overlayer from $t_{\mathrm{NiO}}=0$ (sample a) to $t_{\mathrm{NiO}}=6.5 \mathrm{~nm}$ (sample g). Right: corresponding hysteresis loops taken at $\mathrm{T}=5 \mathrm{~K}$. (C) IOP publishing. Reproduced with permission from [89]. All rights reserved.

The FC/ZFC magnetization curves are reported in fig. 15 . The difference between $M_{\mathrm{FC}}$ and $M_{\mathrm{ZFC}}$ difference decreases upon increasing $T$, because the NP magnetic moment gain enough thermal energy to overcome the barriers due to magnetic anisotropy, and tend to align parallel to $\mathbf{H}$. When $M_{\mathrm{FC}}-M_{\mathrm{ZFC}}=0$, the NPs pass to the SPM (reversible) phase. At the corresponding temperature $T_{\max }$, $M_{\mathrm{ZFC}}$ is maximum, so that $T_{\max }$ can be considered an estimate of $T_{\mathrm{B}}$. However, the behavior of the curves for low values of the shell thickness at high temperatures (200-300 K) suggest that the superparamagnetic NP are coupled by exchange and magnetostatic interactions. Finally, when the $\mathrm{NiO}$ form a film in which the Ni NP cores are embedded, a larger $T_{\max }$ is displayed. The reason of this behavior, in analogy with what observed on $\mathrm{Co} @ \mathrm{CoO} \mathrm{NP}$, is a stronger FM/AF interface coupling, together with a higher magnetic anisotropy, and a blocking temperature $T_{\mathrm{B}}>\mathrm{RT}$, causing a reduction of the effect of the magnetostatic inter-NP interactions. A small EB field, $H_{\text {bias, }}$, is measured up to shell thickness $t_{\mathrm{s}}=1.6 \mathrm{~nm}$, but when $t_{s}$ increases, $H_{\text {bias }}$ is greater, with a steep increase and no sign of saturation (see fig. 16). The maximum value of the EB field is $H_{\text {bias }}=0.57$ kOe. The samples grown with sequential layer deposition have lower $H_{\text {bias }}$ when their shell has a thickness similar to the one obtained for the annealed sample. The differences in the core/shell 
interface quality can be the cause for this result. On the on the other hand, with sequential layer deposition is possible to tune EB by simply adjusting the NiO overlayer thickness, i.e. the shell thickness keeping fixed the NP core diameter.

Figure 16. Evolution of coercition field $H_{c}$, remanence coercition field $H_{c r}$ and bias field $H_{b}$ with the estimated shell thickness from Ni@NiO NP films grown with sequential layer deposition. The open symbols show the results of the same quantities obtained from Ni@NiO NP with comparable size obtained by annealing metallic Ni NP film in air. (C) IOP publishing. Reproduced with permission from [89]. All rights reserved.

As mentioned before, physical synthesis of NP with LPGAS and some auxiliary methods (like evaporation from other sources) allows creation of different species of oxide shells and matrices $[48,82]$. Relevant results were recently obtained in the case of Co@CoO NP (Co core diameter $d \approx$ $5 \mathrm{~nm}$ ) embedded in $\mathrm{NiO}$ matrix [90]. The $\mathrm{CoO}$ shells were obtained during the NP synthesis in the LPGAS, while $\mathrm{NiO}$ matrix (and $\mathrm{Nb}$ for a non magnetic matrix in reference samples) were realized with rf sputtering. SQUID magnetometry measurements revealed an important increase of the blocking temperature $\mathrm{T}_{\mathrm{B}}$ well beyond room temperature, in one case above $\mathrm{T}=400 \mathrm{~K}$, in contrast with the $\mathrm{Co} @ \mathrm{CoO}$ embedded in $\mathrm{Nb}$, where $\mathrm{T}_{\mathrm{B}}$ could reach a maximum value of $70 \mathrm{~K}$. The $\mathrm{Co} @ \mathrm{CoO} / \mathrm{NiO}$ samples also shown a relevant value of $\mu_{0} H_{E}=14 \mathrm{mT}$ and some coercivity at room temperature. The outstanding enhancement of $T_{B}$ is explained as due to a combined magnetic effect involving both $\mathrm{CoO}$ shell and $\mathrm{NiO}$ matrix, in particular the combination of the $\mathrm{CoO}$ high anisotropy and of the high Neel temperature of $\mathrm{NiO}$ (through a proximity effect), providing in this way a sufficiently high AF anisotropy at the core-shell anisotropy to enhance the effective anisotropy of the Co cores. It is worth mentioning some preliminary studies obtained by the "reversed" system, in particular Ni@CoO NP obtained with the sequential layer deposition method, previously described [91]. In situ XPS analysis revealed the existence of an interfacial NiO layer obtained during the growth procedure, at variance with observations on $\mathrm{Ni@MgO} \mathrm{[82]} \mathrm{systems,} \mathrm{synthesized} \mathrm{with} \mathrm{a}$ similar method. A possible reason for this different behavior may lie in the electronegativity of $\mathrm{Mg}$ (1.31 Pauling), which is lower compared to the value for $\mathrm{Ni}$ (1.91 Pauling), so oxidation of $\mathrm{Mg}$ is favored. In a previous work [92] Ni@CoO NPs were synthesized with physical method: evaporation of $\mathrm{Ni}$ and laser ablation of $\mathrm{CoO}$ with condensation by means of $\mathrm{Ar}$ [92], and also in that case a thin interfacial $\mathrm{NiO}$ layer was obtained. The shell around the Ni NPs was composed of $\mathrm{Co}_{3} \mathrm{O}_{4}$. EB was observed up to $T=45 \mathrm{~K}$, a temperature close to the Neèl temperature of $\mathrm{Co}_{3} \mathrm{O}_{4}$. Since the electronegativity values of $\mathrm{Ni}$ and $\mathrm{Co}$ (1.88 Pauling) are very close, it is reasonable to deduce that formation of $\mathrm{NiO}$ can take place, as well as oxidized Co. XMCD data taken at remanence from $\mathrm{Ni} @ \mathrm{CoO} \mathrm{NP}$ films synthesized with sequential layer deposition revealed the existence of a dichroic signal at room temperature, a possible indication of stabilization of the $\mathrm{Ni}$ NP cores in a FM phase [91].

\section{3 "Hard" magnetic NP: the case of $L 1_{0} \mathrm{FePt}$}

An obvious solution to the problem of "beating the superparamagnetic limit" could be the use of materials with high anisotropy constant. The choice of the material is crucial for the realization of the new techniques of recording, like Heat Assisted Magnetic Recording (HAMR) or Energy Assisted Magnetic Recording [93]. An example is the FePt alloy in chemically ordered $\mathrm{L}_{0}$ phase. 
This alloy has a face centred tetragonal structure, with an anisotropy constant $\mathrm{K}=6 \cdot 10^{6} \mathrm{~J} / \mathrm{m}^{3}$, so stable grains of 2.5-3 $\mathrm{nm}$ diameters are conceivable. In practice, several effects occurring at the surface of the NP (lattice distortion due to NP faceting, surface modification due to exposure to atmosphere or interaction with the embedding media etc.) can decrease this value by an order of magnitude. Very often FePt NP are synthesized in the fcc A1 phase, where chemical ordering is disrupted and the material shows soft ferromagnetic behavior [93,94]. Similar properties have been found in CoPt NP [95]. The $\mathrm{L}_{0}$ phase can be obtained by thermal annealing a $\mathrm{T}=450^{\circ} \mathrm{C}$, but some strategies are necessary to prevent NP from sintering and agglomeration, when they are already deposited on a substrate. In some experiments, after having grown the NP with a LPGAS, thermal annealing was performed in flight, by letting the NP beam flowing through an oven [96]. In other works, a careful optimization of the design of the magnetron, the sputtering target and of the parameters governing the sputtering and the NP condensation (sputtering voltage, current density and inert gas pressure) permitted the direct synthesis of monodispersed FePt NP in the $\mathrm{L}_{0}$ phase [93, 97,98] (see fig. 17).

Figure 17. a) HR- TEM image of an octahedral $\mathrm{L} 1_{0} \mathrm{FePt}$ nanoparticle obtained with physical synthesis, with b) corresponding structure model. c) Hysteresis loops obtained at $\mathrm{T}=300 \mathrm{~K}$ and $\mathrm{T}=5 \mathrm{~K}$ from octathedral $\mathrm{L} 1_{0}$ FePt NP assemblies. Reproduced from [97] with permission from John Wiley and Sons.

Another possibility can be the formation of a shell around the NP or an inert matrix during the film growth, in order to avoid sintering $[99,100]$. This method was applied, with partial success by using silica and $\mathrm{MgO}[101,102]$. The necessity of controlling the surface effects, which can modify and degrade the required magnetic properties of FePt NP, has been addressed, and coating or capping with different metals has been seen as a possible solution [103]. This type of fabrication, when applied to physically synthesized NP, can be obtained by the use of multiple sources of evaporation.

\subsection{Core@shell NP realized with multimagnetron approach}

A recent instrumental developments of LPGAS is the planar multimagnetron source, with three targets mounted on the same assembly [50]. Nanoalloy particles and dumb-bell NP can then be realized in one-step procedure inside the source, with great flexibility in varying the alloy relative composition and size. Another important apparatus of new conception is the Multiple Ion Cluster Source (MICS) [47,48,104], which permits direct synthesis of nanoalloy particles, core@shell and core@shell@shell NP. The MICS hosts three independent magnetrons mounted on separate linear drives, so that the magnetrons can be positioned in different regions of the gas aggregation region. In this way, sputtering from different materials and condensation will occur at different distances from the exit aperture of the source (see fig. 17). There is also an additional linear drive for the global positioning of the three magnetrons inside the source, with a considerable increase in the number of parameters controlling the NP growth, and a consequent increase in the complexity of the nanostructures to be fabricated. As an example, results on $\mathrm{Au} @ \mathrm{TiO}_{\mathrm{x}} \mathrm{NP}$ obtained with MICS are reported [48]. This system presents interesting application and may play a fundamental role in photocatalysis, because of the presence of the Au core surface plasmon resonance which allows electron transfer to the $\mathrm{TiO}_{2}$ shell, when the NP is illuminated by visible light. The NP were synthesized obtaining first Au@Ti NP in the source and oxidizing the Ti shell in-flight before the deposition on the substrate, in a similar way used to produce $\mathrm{CeO}_{\mathrm{x}} \mathrm{NP}[43,44]$. 
Figure 18. Sketch of the multiple ion cluster source described in [48]. (a) M1 and M2 are two magnetrons, both in the aggregation zone (AZ). (b) Au or (c) TiOx NPs can be obtained with a single magnetron, while core@shell,Au@TiOx are synthesized by using M1 and M2 at the same time. Reproduced from [48] with permission of the Royal Society of Chemistry

The structure was studied with Cs-corrected STEM and the electronic properties with XPS, revealing the high degree of crystal order in Au core and the amorphous phase of the $\mathrm{TiO}_{\mathrm{x}}$ shell, mainly composed of insulating $\mathrm{TiO}_{2}$.

\section{Low pressure gas phase synthesis of oxide nanoparticles: interplay between structure and functional properties in $\mathrm{CeO}_{2-\mathrm{x}}$.}

Oxide NP play a major role in many fields of advanced technology: photocatalysis, energy conversion and storage, biomedicine and memories are just some examples. Physical synthesis of oxide nanoclusters with LPGAS from oxide targets may present some difficulties or it can be impossible, if DC sputtering is used, because of the intrinsic insulating nature of many of these materials. An alternative can be represented by the use of RF magnetron plasma sputtering [105]. Another possibility is the use of a low energy cluster beam deposition (LECBD) apparatus described in [106,107]. This system makes use of a ND:Yag laser for vaporization from a target and of a He condensation system, producing supersonic beams of clusters. With this technique, quasiperfect $\mathrm{ZnO} \mathrm{NP}$ (with diameters $d \approx 6 \mathrm{~nm}$ ) were produced, with a very good degree of stoichiometry. Additional incorporation of atomic oxygen during the cluster growth improved the degree of crystallinity in the wurtzite phase and removed native defects. ZnO NP presented also interesting properties of organization when deposited. In particular, the oriented attachment process was observed, with formation of large crystalline domains, envisaging fascinating perspective in the growth of mesostructures and superarchitectures from smaller nanocrystals [107]. Another example of techniques for the synthesis of oxide NP is Pulsed Laser Vaporization, which was extensively used for growth of $\mathrm{TiO}_{\mathrm{x}}$ NP [108]. In particular, with careful choice of the PLV working parameters (laser fluence and background pressure) it was possible to obtain "black" $\mathrm{TiO}_{2} \mathrm{NP}$, with a crystalline rutile core and a disordered $\mathrm{Ti}_{2} \mathrm{O}_{3}$ shells, which can in principle exhibit higher photocatalytic activity with respect to ordinary white $\mathrm{TiO}_{2}$ [108].

An alternative way to produce oxide NP by using DC magnetron sputtering from metallic target is to oxidize in-flight the metal NP, either inside the gas aggregation region, or immediately after their exit from the source, in a separate chamber, before deposition on the substrate. This method can be very effective for reactive metals, like for instance rare earths [43, 44]. Among the rare earth oxides, certainly $\mathrm{CeO}_{\mathrm{x}}$ is one of the most studied, because its capability of storing and releasing oxygen atoms as a reducible oxide, by switching reversibly between two stable oxidation states, corresponding to formation $\mathrm{CeO}_{2}$ and $\mathrm{Ce}_{2} \mathrm{O}_{3}$ compounds. This property can be utilized in many areas of application: catalysis, energy conversion and storage, biomedicine and memories [109]. Since the redox process is reversible, there are great implications in phenomena related with oxygen transport within the material. For this reason, a huge amount of studies have been carried out on Ceria in different forms [109]. In the last decade, great attention was devoted to the influence of reduced dimensionality in its redox properties, focusing on $\mathrm{CeO}_{\mathrm{x}}$ ultrathin epitaxial films and nanostructures [110]. It was found that in cerium oxide ultrathin films and NP a higher surface-tobulk ratio can promote an increased the degree of reducibility [111-113]. Other factors playing a major role in this increase are the modifications of the electronic structure and a more pronounced mobility of the oxygen ions. Also, when nanocystalline cerium oxide supports metal NP, the energy of formation of oxygen vacancies is highly reduced with respect to the case of large surfaces, so 
oxygen can be transferred more easily to the metal NP [114]. Nanostructured ceria was previously obtained by magnetron sputtering and inert gas condensation, with a resulting high degree of nonstoichiometry [115-117] because of the presence of a massive amount of $\mathrm{Ce}^{3+}$ ions. In more recent works, a LPGAS with magnetron sputtering from pure Ce metal target was used, and a systematic study of the effects of NP size and of the different procedures of oxidation (in-flight and postdeposition) on the structure and on reducibility has been carried out $[43,44]$. In particular three different samples were prepared with the same NP size distribution $(<\mathrm{d}>=8.5 \mathrm{~nm}$ with a size dispersion $\Delta \mathrm{d}=3.9 \mathrm{~nm}$ ) [44]:

1) In the first sample, the ceria NP assemblies were obtained by deposition of metal NP followed by exposure to $\mathrm{O}_{2}$ ("post-oxidized sample").

2) A second film was realized by obtaining oxidized Ce NP in the aggregation region of the source, using a mixture of $\mathrm{Ar}$ and $\mathrm{O}_{2}$ as gas carrier ("directly oxidized sample").

3 ) In the last case metal Ce NP were deposited on the support, and they were exposed to residual $\mathrm{O}_{2}$ gas. The NP were oxidized after a short time because of the high reactivity of Ce ("sample oxidized in HV").

The supports used were $\mathrm{Si} / \mathrm{SiO}_{\mathrm{x}}$ for the samples measured with XPS, and lacey grids for electron microscopy analysis. Fig. 19 shows STEM images of the NP obtained with the three different procedures. The "post oxidized" NP present a $\mathrm{CaF}_{2}$ crystalline structure; the faces of the nanocrystal exhibit mainly a (111) and a (100) orientation. The "directly oxidized" NP (see Figure 19b) have an irregular shape, and a detailed HR-TEM analysis demonstrated that they are mainly polycrystalline. The STEM images taken from the NP "oxidized in HV" (Fig. 19 c) were taken with a higher magnification compared to the previous cases. Although some NP agglomerates are visible, the structure of the single NP is similar to the "post oxidized NP".

Figure 19. STEM images of $\mathrm{CeO}_{2-x}$ NP. (a) post oxidized NP, (b) directly oxidized NP, (c) NP oxidized in HV. Insets: high-resolution HAADF images (C IOP publishing. Reproduced with permission from [44]. All rights reserved.

By means of Electron Energy Loss Spectroscoy (EELS)-STEM maps of the NP it was possible to obtain the distribution of $\mathrm{Ce}^{3+}$ and $\mathrm{Ce}^{4+}$ inside the single NP. This analysis was performed by performing a point-by-point analysis of the Ce $\mathrm{M}_{4,5}$ edge EELS spectra. For the "post oxidized sample" (Fig. 20a), $\mathrm{Ce}^{3+}$ ions are located mainly in the surface region of the NP, as they form a shell of thickness $t \approx 0.7 \mathrm{~nm}$, probably because of exposure to atmosphere while moving the samples from the source chamber to TEM, because of adsorption of $\mathrm{OH}$ groups. In the directly oxidized NP (fig. 20b), the presence of $\mathrm{Ce}^{3+}$ ions is significant also in the core. The explanation can lie in the different procedure used to obtain the NP: during the formation of the NP in the gas aggregation region, $\mathrm{CeO}_{2}$ clusters with $\mathrm{Ce}^{3+}$ are formed at the surface, interacting with each other and coagulating into bigger NP; the process of coagulation gives as a result the presence of $\mathrm{Ce}^{3+}$ ions also in the NP core, at the borders between the original smaller clusters, which now form grains. The EELS-STEM map reported from the sample oxidized in HV (figure 20c) shows instead an agglomerate of NP with $\mathrm{Ce}^{3+}$ and $\mathrm{Ce}^{4+}$ ion distribution within the single NP similar to the case of figure $20 \mathrm{a}$. 
Figure 20. EELS maps of the distribution of $\mathrm{Ce} 3+$ (red) and $\mathrm{Ce} 4+$ (green) ions, obtained with a fitting of $\mathrm{Ce}$ $\mathrm{M}_{4,5}$ reference spectra from $\mathrm{Ce} 3+$ and $\mathrm{Ce} 4+$ : a) post oxidized $\mathrm{NP}$, b) directly oxidized $\mathrm{NP}$, and c) NP oxidized in HV. (C IOP publishing. Reproduced with permission from [44]. All rights reserved.

The three types of samples were all transferred quickly to a different UHV chamber and treated with a reduction process, which was carried out by thermal annealing in UHV at increasing temperature (from RT to $\mathrm{T}=1020 \mathrm{~K}$ with steps of $250 \mathrm{~K}$ ), and with an oxidation process by annealing at $\mathrm{T}=1020 \mathrm{~K}$ in presence of oxygen. The behavior of NPs in reducing and oxidizing conditions was monitored with XPS from Ce 3d core levels during the reduction-oxidation cycles and an example is shown in figure 21a) [43,44].

Figure 21. a) Ce $3 d$ XPS spectra from a ceria NP sample. Spectra (A)-(D) were acquired at RT, and after different annealing steps in UHV at $520 \mathrm{~K}, 770 \mathrm{~K}$ and $1020 \mathrm{~K}$; spectrum $\mathrm{E}$ was acquired after annealing in $\mathrm{O}_{2}$ at $1020 \mathrm{~K}$. The main Ce3+-related features are indicated with a red arrow. b) Evolution of $\mathrm{Ce}^{+}$ concentration obtained by XPS Ce $3 \mathrm{~d}$ core-level analysis. Left side: $\mathrm{Ce} 3^{+}$concentration from samples obtained from XPS analysis of the as-deposited samples in the NP source chamber. Right side: $\mathrm{Ce}^{+}$ concentration from XPS data taken in the second UHV chamber after different steps during reduction and oxidation. (C) IOP publishing. Reproduced with permission from [44]. All rights reserved.

The analysis was performed by fitting the Ce $3 \mathrm{~d}$ core level spectra with a linear combination of the $\mathrm{Ce}^{3+}$ and $\mathrm{Ce}^{4+}$ reference spectra [43]. A comparison of the results from the three samples (figure 21 b) demonstrated that the sample oxidized in HV is generally more reduced than the other two. Moreover, it was not possible to re-oxidize the NP thoroughly. Instead, the other two samples could be completely oxidized. The trend of the $\mathrm{Ce}^{3+}$ concentration vs. the annealing temperature in the directly oxidized sample is similar to the one of the sample oxidized in $\mathrm{HV}$, but its reduction is not complete. By combining these results with the ones obtained by the TEM analysis, we can relate the reduction-oxidation properties of these three types of NP with their structure. The post oxidized NP with their regular crystalline structure have probably a high mobility of oxygen ions. Also, the (100) exposed planes visible in the TEM images have energy of formation of $\mathrm{O}$ vacancies lower than the one of (111) planes, and all these factors can be responsible for the high reducibility of such NP films. At variance, the directly oxidized NP show a polycrystalline structure, resulting in irregularities and defects especially at the borders of their grains and this presence can hamper the mobility of $\mathrm{O}$ ions. Regarding the NP oxidized in HV, their tendency to form agglomerates can be explained by their high degree of reducibility. These agglomerates can hamper a complete reoxidation, resulting in a lower catalytic efficiency for ceria NP realized in this way.

The behavior of ceria NP of different sizes $(<\mathrm{d}>=6 \mathrm{~nm}, 9 \mathrm{~nm}$ and $14 \mathrm{~nm})$ was also analyzed. In order to investigate the (100) surface contribution to the reducibility we compared the results obtained from the NP with $<\mathrm{d}>=9 \mathrm{~nm}$ with the ones obtained on epitaxial ceria films grown by reactive evaporation of $\mathrm{Ce}$ in $\mathrm{UHV}$ on $\mathrm{Pt}(111)$ surface [43]. The exposed film surface corresponds to the (111) plane. The film growth conditions have been chosen in order to have the same surface to volume ratio as the $9 \mathrm{~nm}$ NP sample. By XPS analysis during oxidation and reduction cycle, we observed a stronger reducibility for NP, possibly because of the fact that exposed surface is (100) which is more favorable to oxygen release, instead of the (111) surface like in the case of the film. 
The high reducibility of NP may have important implications for their catalytic efficiency. In summary, these studies are relevant for the comprehension of the mechanism of nanocatalysis, and may have some important implications in production of catalytic NP.

\section{Conclusions.}

Some selected examples of experimental activity on low pressure gas phase synthesis metal@oxide, core@shell and oxide NP have been reviewed. In particular, significant effort have been put in trying to explain the physical principles of working of the LPGAS, relating them with the properties of the produced NP, and showing some strength points of the bottom-up physical synthesis methods: one-step synthesis procedure, absence of unwanted chemical by-products and ligand, and higher degree of control of the NP characteristics with respect to the widely used and more economic chemical routes, that can alter the intrinsic properties of "model system" NP. Among the many issues which can be of relevance in nanotechnology products, stabilization of magnetic NP and basic redox properties of Ceria nanostructures have been reviewed, taking also in consideration the possibilities of more complex NP architectures offered by new instrumental developments, like co-deposition and multimagnetron approach, which can be of significant help in striding towards new functionalities and rational design in nanoscience and nanotechnology.

\section{ACKNOWLEDGEMENTS}

The work was partially supported by the Italian MIUR under grant FIRB RBAP115AYN (Oxides at the nanoscale: multifunctionality and applications) and by the regional project "HEGOS - nuove pompe di calore per l'Harvesting EnerGeticO in Smart buildings", POR FESR 2014-2020, Azione 1.2.2. Some of the activity was performed within the COST Action CM1104 "Reducible oxide chemistry, structure and functions".

\section{References}

[1] Parak W J, Manna L, Simmel F C, Gerion D and Alivisatos P 2004 Quantum Dots, in Nanoparticles, ed. by Schmidt G, (Weinheim; Wiley-VCH).

[2] Bourzac K 2013 Nature 493283

[3] Shirasaki Y, Supran G J, Bawendi M G and Bulovic V 2013 Nature Phot. 713

[4] Smith A M and Nie S 2004 Analyst 129672

[5] Gao X, Cui Y, Levenson R M, Chung L W K and Nie S 2004, Nature Biotechnology 22969

[6] Duncan T V 2011 Journal of Colloid and Interface Science 3631

[7] Metak A M, Nabani F and Connolly S N 2015 LWT-Food Science and Technology 64781

[8] Blasiak B, van Veggel F C J M and Tomanek B 2013 Journal of Nanomaterials 2013148578

[9] Na H B, Song I C and Hyeon T 2009 Adv. Mat. 212133

[10] Martin T P 1996 Phys. Rep. 273199

[11] Johnston R L. 2012 in Metal Nanoparticles and Alloys, ed. By Johnston R L and Wilcoxon, Frontiers in Nanoscience vol. 3 (Elsevier)

[12] Marks L D 1994 Rep. Prog. Phys. 57603

[13] Reinhard D., Hall B D, Ugarte D and Monot R 1997 Phys. Rev. B 557868

[14] Wang R, Dmitrieva O, Farle M, Dumpich G, Acet M, Mjia-Rosales S, Perez-Tijerina E, Yacaman M J and Kisielowski C 2009 J. Phys. Chem. C 1134395

[15] D'Addato S, Grillo V, Tondi R, Valeri S and Frabboni S 2011 J. Phys.: Condensed Matter 23 175003 
[16] Barke I, Hartmann H, Rupp D et al. 2015 Nature Commun. 66187

[17] Binns C, Qureshi M T, Peddis D, Baker S H, Howes P B, Boatwright A, Cavill S A, Dhesi S S, Lari L, Kröger R and Langridge S 2013 Nano Lett. 133334.

[18] Spadaro M C, Fazio E, Neri F, Trusso S and Ossi P M 2015 EPL 10925002

[19] Spadaro M C, Fazio E, Neri F, Ossi P M and Trusso S 2014 Appl. Phys. A 117137

[20] De Toro J A, Normile P S and Binns C 2017 Types of Cluster Sources, in Gas-Phase Synthesis of Nanoparticles, ed. by Huttel, Y (Weinheim, Wiley-VCH)

[21] Strobel R and Pratsinis S E 2007 J. Mater. Chem. 174743.

[22] Kortshagen U R, Sankaran R M, Pereira R N, Girshick S L, Wu J J and Aydil E S 2016

Chem. Rev. 116, 11061

[23] Mariotti D and Sankaran R M 2010 J. Phys. D: Appl. Phys. 43323001

[24] Mangolini L 2017 J. Phy. D: Appl. Phys. 50373003

[25] Kumar A, Kang S, Larriba-Andaluz C, Ouyang H, Hogan C J and Sankaran R M, Nanotechnology 201425385601

[26] Hunter K I, Held J T,. Mkhoyan K A and Kortshagen U R 2017 ACS Appl. Mat. \& Int. 9

8263

[27] Feng J, Biskos G and Schmidt-Ott A 2015 Sci. Rep. 515788

[28] Sattler K, Mühlbach J, Recknagel E 1980 Phys. Rev. Lett. 45821

[29] Binns C 2001 Surf. Sci. Rep. 441

[30] Pettiette C L, Yang S H, Craycraft M J, Cociecao J, Laaksonen R T, Chesnowsky O and Smalley R E 1988 J. Chem. Phys. 885377

[31] Marzin J-I, Gérard J-M, Izraël A, Barrier D and Bastard G 1994 Phys. Rev. Lett. 73716

[32] Luches P, Pagliuca F, Valeri S, Illas F, Preda G and Pacchioni G 2012 J. Phys. Chem. C 116 1122

[33] Benedetti F, Luches P, Spadaro M C, Gasperi G, D’Addato S, Valeri S and Boscherini F 2015 J. Phys. Chem. C 1196024

[34] Haberland H, Karrais M and Mall M 1990 MRS Proc. 206291

[35] Haberland H, Karrais M, Mall M and Thurner Y 1992 J. Vac. Sci. Technol. A 103266

[36] Haberland H 2017, Hystory, Some Basics, and an Outlook, in Gas-Phase Synthesis of

Nanoparticles, ed. by Huttel, Y (Weinheim, Wiley-VCH)

[37] Smirnov B M 2000 Clusters and Small Particles in Gases and Plasmas (New York, Springer Verlag)

[38] Smirnov B M, Shyjumon I and Hippler R 2007 Phys. Rev. E 75066402

[39] Kesälä E, Kuronen A and Nordlund K 2007 Phys. Rev. B 75174121

[40] Quesnel E, Pauliac-Vaujour E and Muffato V 2010 J. Appl. Phys. 107054309

[41] Briehl B, Urbassek H M 1999 J. Vac. Sci. Technol. A 17256

[42] Granqvist C G and Buhrman R A 1976 J. Appl. Phys. 472200

[43] Spadaro M C, D’Addato S, Gasperi G, Benedetti F, Luches P, Grillo V, Bertoni G and Valeri S 2015 Beilstein J. Nanotechnol. 660

[44] Spadaro M C, Luches P, Bertoni G, Grillo V, Turner S, Van Tendeloo G, Valeri S and D’Addato S 2016 Nanotechnology 27425705

[45] Haberland H, Insepov Z and Moseler M 1995 Phys. Rev. B 5111061

[46] D'Addato S, Gragnaniello L, Valeri S, Rota A, di Bona A, Spizzo F, Panozaqi T and Schifano S F 2010 J. Appl. Phys. 107104318

[47] Llamosa D, Ruano M, Martínez L, Mayoral A, Roman E, Garcia-Hernández M and Huttel Y 2014 Nanoscale 613483

[48] Martínez L, Mayoral A, Espiñeira M, Roman E, Palomares F J and Huttel Y 2017 Nanoscale 9 6463

[49] Benelmekki M, Vernieres J, Kim J H, Diaz R E, Grammatikopolous P and Sowwan M 2015 Mater. Chem. Phys. 151275 
[50] Johnson G E and Laskin J 2017 In-Plane Multimagnetron Approach, in Gas-Phase Synthesis of Nanoparticles, ed. by Huttel, Y (Weinheim, Wiley-VCH)

[51] de Heer WA 1993 Rev. Mod. Phys. 65611

[52] Binns C, Trohidou K. N, Bansmannm J, Baker S H, Blackman J A, Bucher J-P, Kechrakos, D, Kleibert A, Louch S, Meiwes-Broer K-H, Pastor G M, Perez A, Xie Y 2005 J. Phys. D: Appl. Phys. 38 R357.

[53] Huttel Y (ed.) 2017 Gas-Phase Synthesis of Nanoparticles, (Weinheim, Wiley-VCH)

[54] Vystavel T, Palasantzas G, Koch S A and De Hosson J Th M 2003 Appl. Phys. Lett. 82197

[55] Koch S A, Palasantzas G, Vystavel T, De Hosson J Th M, Binns C and Louch S 2005 Phys Rev. B 71085410

[56] de La Presa P, Rueda T., Hernando A, Ramallo-López J M, Giovanetti L J and Requejo F G 2008 J. Appl. Phys. 103103909

[57] Kleibert A, Bulut F, Gebhardt R K, Rosellen W, Sudfeld D, Passig J, Bansmann J, MeiwesBroer K H and Getzlaff M 2008 J. Phys.: Condens. Matter 20445005

[58] Holloway P H and Hudson J B 1974 Surf. Sci. 43123

[59] Cornish A, Eralp T, Shavorskij A, Bennett R A, Held G, Cavill S A, Potenza A, Marchetto H and Dhesi S S 2010 Phys Rev B 81085403

[60] Pratt A, Lari L, Hovorka O, Shah A, Woffinden C, Tear S P, Binns C and Kröger R 2014 Nat. Mat. 1326

[61] Cabrera N and Mott N F 1949 Rep. Prog. Phys. 12163

[62] Wang C M, Baer D R, Thomas L E and Amonette J E, Antony J, Qiang Y and Duscher G 2005

J. Appl. Phys. 98094308

[63] Jiles D 1998 Magnetism and Magnetic Materials (London: Chapman and Hall)

[64] Kittel C 1946 Phys. Rev. 70965

[65] Dormann J L, Fiorani D and Tronc E 1997 Adv. Chem. Phys. 98283

[66] D'Addato S 2017 In-Flight Analysis, in Gas-Phase Synthesis of Nanoparticles, ed. by Huttel, Y (Weinheim, Wiley-VCH)

[67] Cox D M, Trevor D J, Whetten R L, Rohlfing E A and Kaldor A 1985 Phys. Rev. B 327290

[68] Bucher J P, Douglass D C and Bloomfield L A 1991 Phys. Rev. Lett. 663052

[69] Billas I M L, Becker J A, Châtelain A and de Heer W A 1993 Phys. Rev. Lett. 714067

[70] Billas, I M L, Châtelain A and de Heer W A 1997 J. Magn. Magn. Mat. 16864

[71] Payne F W, Jiang W, Emmert J W, Deng J and Bloomfield L A 2007 Phys. Rev. B 75094431

[72] Xu, X, Yin S, Moro R and de Heer W A 2008 Phys. Rev. B 78054430

[73] Xu, X, Yin S, Moro R, Liang A, Bowlan J and de Heer W A 2011 Phys. Rev. Lett. 107057203

[74] van Dijk C, Bowlan J, de Heer W A, Rasing Th and Kirikyuk A 2015 J. Phys. Chem. C 119 11153

[75] Knickelbein M B 2007 Phys. Rev. B 75014401

[76] Peredkov S, Neeb M, Eberhardt W, Meyer J, Tombers M, Kampshulte H and NiednerSchatteburg, G 2011 Phys. Rev. Lett., 107233401

[77] Niemeyer M, Hirsch K, Zamudio-Bayer V, Langenberg A, Vogel M, Kossick M, Ebrecht C, Egashira K, Terasaki A, Möller T, Issendorff B v and Lau J T 2012 Phys. Rev. Lett. 108057201

[78] Langegnberg A, Hirsch K, Lavicki A, Zamudio-Bayer V, Niemeyer M, Chmiela P, Langbehn, B, Terasaki A, Issendorff B v and Lau J T 2014 Phys. Rev. B 90184420

[79] Weller D and Moser A 1999 IEEE Trans. Magn. 1999354423

[80] Nogués J, Sort J, Langlais V, Skumryiev V, Suriñach S, Muñoz J S and Baró M D 2005 Phys. Rep. 42265

[81] D'Addato S, Grillo V, Altieri S., Frabboni S and Valeri S 2012 Appl. Surf. Sci. 26013

[82] D'Addato S, Grillo V, Altieri S, Frabboni S, Rossi F and Valeri S 2011 J. Phys. Chem C 115 14044

[83] Skumryev V, Stoyanov S, Zhang Y, Hadjipanayis G, Givord D and Nogués J 2003 Nature 423 850 
[84] Nogués J, Skumryiev V, Sort J, Stoyanov S and Givord D 2006 Phys. Rev. Lett. 97157203

[85] Seto T, Akinaga H, Takano F, Koga K, Orii T and Hirasawa M 2005 J. Chem. Phys. B 109 13403

[86] Zhou Y Z, Chen J S, Tay B K, Hu J F, Chow G M, Liu T and Yang P 2007 Appl. Phys. Lett. 90 043111

[87] Johnston-Peck A C, Wang J and Tracy J B 2009 ACS Nano 31077

[88] D'Addato S, Spadaro M C, Luches P, Grillo V, Frabboni S, Valeri S, Ferretti A M, Capetti E and Ponti A 2014 Appl. Surf. Sci. 3062

[89] Spadaro M C, D'Addato S, Luches P, Valeri S, Grillo V, Rotunno E, Roldan M A, Pennycook S J, Ferretti A M, Capetti E and Ponti A 2015 Nanotechnology 26405704

[90] De Toro J A, Marques D P, Muñiz P, Skumryiev V, Sort J, Givord D and Nogués J 2015 Phys. Rev. Lett. 115057201

[91] Spadaro M C, Luches P, Benedetti F, Valeri S, Turchini S, Bertoni G, Ferretti A M, Capetti E, Ponti A and D'Addato S. 2017 Appl. Surf. Sci. 3961860

[92] Ceylan A, Rumaiz A K and Sha I 2007 J. Appl. Phys. 101094302

[93] Wang J-P 2008 Proc. IEEE 961847

[94] Sun S H, Murray C B, Weller D, Folks L and Moser A 2000 Science 2871989

[95] Alloyeau D, Ricolleau C, Mottet C, Oikawa T, Langlois C, Le Bouar Y, Braidy N and Loiseau A 2009 Nature Mat. 8940

[96] Dmitrieva O, Rellinghaus B, Kästenr J and Dumpich G 2007 J. Cryst. Growth 303645

[97] Qiu J-M and Wang J-P 2007 Adv. Mater. 191703

[98] Akdogan O, Li W, Hadjipanays G C, Skomski R and Sellmyer D J 2012 J. Appl. Phys. 111 $07 \mathrm{~B} 535$

[99] Liu C, Wu X, Klemmer T, Shukla N, Weller D, Roy A G, Tanase M and Laughin D 2005 Chem. Mat. 17620

[100] Tornou A, Panagloiotopoulos I, Gournis D and Kooi B 2007 J. Appl. Phys. 102023910

[101] Colak L and Hadjipanais G 2009 IEEE Trans. Magn. 454081

[102] D’Addato S, Grillo V, di Bona A, Luches P, Frabboni S, Valeri S, Lupo P, Casoli F and Albertini F 2013 Nanotechnology 24495703

[103] Antoniak C, Gruner M E, Spasova M, Trunova A V, Römer F M, Warland A, Krumme B, Fauth K, Sun S H, Entel P, Farle M and Wende H 2011 Nature Comm. 2528

[104] Martinez L 2017 Adjustable Multimagnetron Approach in Gas-Phase Synthesis of

Nanoparticles, ed. by Huttel, Y (Weinheim, Wiley-VCH)

[105] Pratontep S, Carroll S J, Xirouchaki C, Streun M and Palmer R E 2005 Rev. Sci. Instrum. 76 045103

[106] Tainoff D, Masenelli B, Boisron O, Guiraid G and Mélinon P 2008 J. Phys. Chem. C 112 12623

[107]Hapiuk D, Masenelli B, Masenelli-Varlot K, Tainoff D, Boisron O, Albin C and Mélinon P 2013 J. Phys. Chem. C 11710220

[108] Tian M., Mahjouri-Samani M, Eres G, Sachan R, Yoon M, Chisholm M F, Wang K, Puretzky A A, Rouleau C M, Geohegan D B and Duscher G 2015 ACS Nano 910482

[109] Trovarelli A and Fornasiero P 2013, Catalysis by Ceria and Related Materials, in Catalytic Science, 2nd edn. Imperial College Pr., London

[110] Luches P and D'Addato S 2016 Reducible Oxide as Ultrathin Epitaxial Films in Springer Series in Material Science 234119

[111] Waser R, Dittmann R, Staikov G and Szot K 2009 Adv. Mater. 212632

[112] Migani A, Vayssilov G N, Bromley S T, Illas F and Neyman K M 2010 J. Mater. Chem. 46 10535

[113] Cafun J D, Kvashnina K O, Casals E, Puntes V F and Glatzel P 2013 Acs Nano 710726

[114] Vayssilov G N, Lykhach Y, Migani A, Staudt T, Petrova G P, Tsud N, Skala T, Bruix A, Illas

F, Prince K C, Matolin V, Neyman K M and Libuda J 2011 Nature Mater. 10310 
[115] Tschöpe A and Ying J Y 1994 Nanostruct. Mater. 4617

[116] Tschöpe A, Liu W, Flytzani-Stephanopoulos and Ying J Y 1995 J. Catal. 15742

[117] Tschöpe A, Schaadt D, Birringer R and Ying J Y 1997 Nanostruct. Mater. 9423 